\title{
Fracture prevention by screening for high fracture risk: a systematic review and meta-analysis
}

\author{
T. Merlijn ${ }^{1}$ - K.M.A. Swart ${ }^{1}$ - H.E. van der Horst ${ }^{1}$ • J.C. Netelenbos ${ }^{2}$ • P.J.M. Elders ${ }^{1}$
}

Received: 26 August 2019 / Accepted: 5 November 2019/Published online: 14 December 2019

(C) The Author(s) 2019

\begin{abstract}
This systematic review and meta-analysis showed a significant reduction of (major) osteoporotic fractures and hip fractures after screening using fracture risk assessment and bone densitometry compared with usual care. The results indicate that screening is effective for fracture risk reduction, especially hip fractures. To perform a systematic review and meta-analysis of population screening for high fracture risk on fracture prevention compared with usual care. MEDLINE and Embase were searched for studies published until June 20th 2019. Randomized studies were selected that screened for high fracture risk using at least bone densitometry, screened in a general population, provided subsequent treatment with anti-osteoporosis medication, had a usual care group as comparator, and had at least one fracture-related outcome (all fractures, (major) osteoporotic fractures, or hip fractures). The primary assessment was the hazard ratio (HR) for fracture-related outcomes. All-cause mortality was a secondary outcome. Random-effects models were used to estimate pooled HRs. We identified 1186 potentially eligible articles and included three randomized studies: the ROSE study, the SCOOP study, and the SOS with a total number of $N=42,009$ participants. Respectively, $11 \%, 15 \%$, and $18 \%$ of the participants in the intervention group started medication. Meta-analysis showed a statistically significant and clinically relevant reduction of osteoporotic fractures $(\mathrm{HR}=0.95,95 \%$ confidence interval $(\mathrm{CI})=0.89-1.00)$, major osteoporotic fractures $(\mathrm{HR}=0.91 ; 95 \% \mathrm{CI}=0.84-0.98)$, and hip fractures $(\mathrm{HR}=0.80 ; 95 \% \mathrm{CI}=0.71-0.91)$, but no reduction of all fractures $(\mathrm{HR}=0.95 ; 95 \% \mathrm{CI}=0.89-1.02)$. The pooled $\mathrm{HR}$ for the secondary outcome all-cause mortality was $1.04(95 \% \mathrm{CI}=0.95-1.14)$. Numbers needed to screen to prevent one fracture were 247 and 272 for osteoporotic fractures and hip fractures, respectively (corresponding to 113 and 124 performed bone densitometry examinations, and 25 and 28 persons being treated). This meta-analysis showed that population screening is effective to reduce osteoporotic fractures and hip fractures. Implementation of screening in older women should be considered as serious option to prevent osteoporotic fractures, especially hip fractures.
\end{abstract}

Keywords Fracture prevention $\cdot$ Fracture risk assessment $\cdot$ Meta-analysis $\cdot$ Osteoporosis $\cdot$ Screening

\section{Introduction}

The ultimate goal of osteoporosis care is to reduce fractures and its consequences, such as disability and pain. There is discussion about how to decrease the current treatment gap,

T. Merlijn and K.M.A. Swart contributed equally to this work.

P.J.M. Elders

p.elders@amsterdamumc.nl

1 Amsterdam UMC, Vrije Universiteit Amsterdam, Department of General Practice and Elderly Care Medicine, Amsterdam Public Health Research Institute, 1081 BT Amsterdam, Netherlands

2 Amsterdam UMC, Vrije Universiteit Amsterdam, Department of Internal Medicine, Endocrine Section, 1081

HV Amsterdam, Netherlands i.e., the fact that a large and increasing proportion of men and women at high-risk for fractures do not receive antiosteoporosis medication [1,2]. Although anti-osteoporosis medication has been proven to be effective in placebocontrolled trials $[3,4]$, and tools are available to identify persons at high-risk [5], it is not clear whether screening for high fracture risk in the general population is effective in reducing fractures.

Different policies exists between countries with respect to population screening for high fracture risk. For instance, the Canadian guideline recommends bone mineral density measurements of all men and women of 65 years and older [6]. In the USA, this applies to all women [7]. However, in the UK and the Netherlands, screening has not been implemented so far, because of the lack of evidence of its effectiveness [8,9]. 
Several large randomized screening studies for fracture prevention have been finished recently [10-12]. None of the studies observed a statistically significant reduction in osteoporotic fractures or major osteoporotic fractures. One study observed a reduction of hip fractures in the screening group compared with usual care [12], but this observation was not confirmed in the other trials $[10,11]$. A meta-analysis of the findings of these trials is needed to determine the effect of population screening for fracture prevention.

We, therefore, performed a systematic review and metaanalysis of randomized controlled trials that have studied the effect of population screening for high fracture risk and subsequent treatment compared with usual care on all fractures, osteoporotic fractures, major osteoporotic fractures, and hip fractures. We hypothesized that screening for fracture risk is effective in reducing fractures.

\section{Methods}

A systematic literature search until June 20th 2019 was performed to identify all screening trials that were published in MEDLINE and Embase (Table 1 for search strategy). Title and abstract (first step) and the full articles (second step) were considered for relevance and eligibility by two independent investigators (TM, KMAS).

Original studies were selected if the study design was a randomized trial in a general population that used at least bone densitometry as screening instrument and subsequent treatment with anti-osteoporosis medication, defined as bisphosphonates, denosumab, or strontium ranelate. Studies were considered if fractures were the outcome, and a usual care group was used as the comparator. The reference lists of selected articles were checked manually for additional studies. Discrepancies between investigators were re-evaluated until consensus was reached. For each included study, data were extracted about design, population, participation, adherence, outcomes, and summary estimates using a standardized form by the two investigators, independently. Risk of bias was assessed with the Cochrane risk of bias tool [13]. The primary outcomes of the meta-analysis included all fractures, (major) osteoporotic fractures, and hip fractures. Osteoporotic

Table 1 Literature search strategy in MEDLINE

\section{Search strategy}

(Mass Screening [MeSH] AND (Fractures, Bone [MeSH] OR

Osteoporosis [MeSH] or osteoporotic fractures [MeSH]) AND Randomized Controlled Trial [MeSH])

OR

((screening [tiab] OR screen* [tiab]) AND (osteoporosis [tiab] OR fractures [tiab] OR osteoporo* [tiab]) AND (randomized [tiab] OR randomized [tiab] OR randomly [tiab] OR trial [tiab])) fractures were defined as all fractures except for skull, finger, hand, toe, and foot fractures. Major osteoporotic fractures were defined as all hip, vertebral, wrist, and humerus fractures. All-cause mortality was a secondary outcome. The hazard ratio (HR) was the principal summary measure. Betweenstudy heterogeneity was estimated using $I^{2}$-statistics. Results were pooled if the studies were sufficiently homogenous $\left(I^{2}<\right.$ $50 \%$ ), using a random effects model. In post-hoc sensitivity analysis, the hip fracture data of one study were excluded to examine if the findings were driven by this study. The number needed to screen and number needed to treat were calculated as the inverse of the absolute risk difference of participants in the usual care versus the screening group who completed a questionnaire or who started anti-osteoporosis medication, respectively. The analyses were performed in Review Manager 5.3. The statistical significance level was set at a two-tailed alpha level of 0.05 .

\section{Results}

The literature search resulted in 1186 articles (Fig 1). Nine articles reported trials on screening for fracture prevention, of which three were eligible for inclusion. Reasons for exclusion were the lack of a usual care group [14, 15]; dual-energy $\mathrm{x}$-ray absorptiometry (DXA) was not part of screening [16, 17], the use of medication that is no longer used for the treatment of osteoporosis (hormone replacement therapy) [18], or the lack of follow-up data [19]. The included studies were the ROSE study, the SCOOP study, and the SOS [10-12].

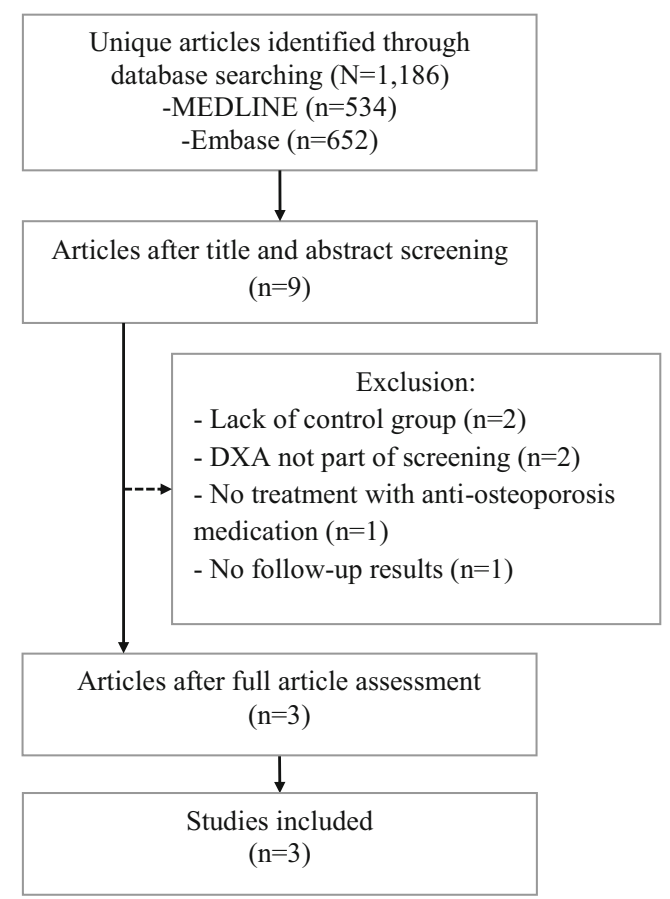

Fig 1 Flow of information through selection procedure 


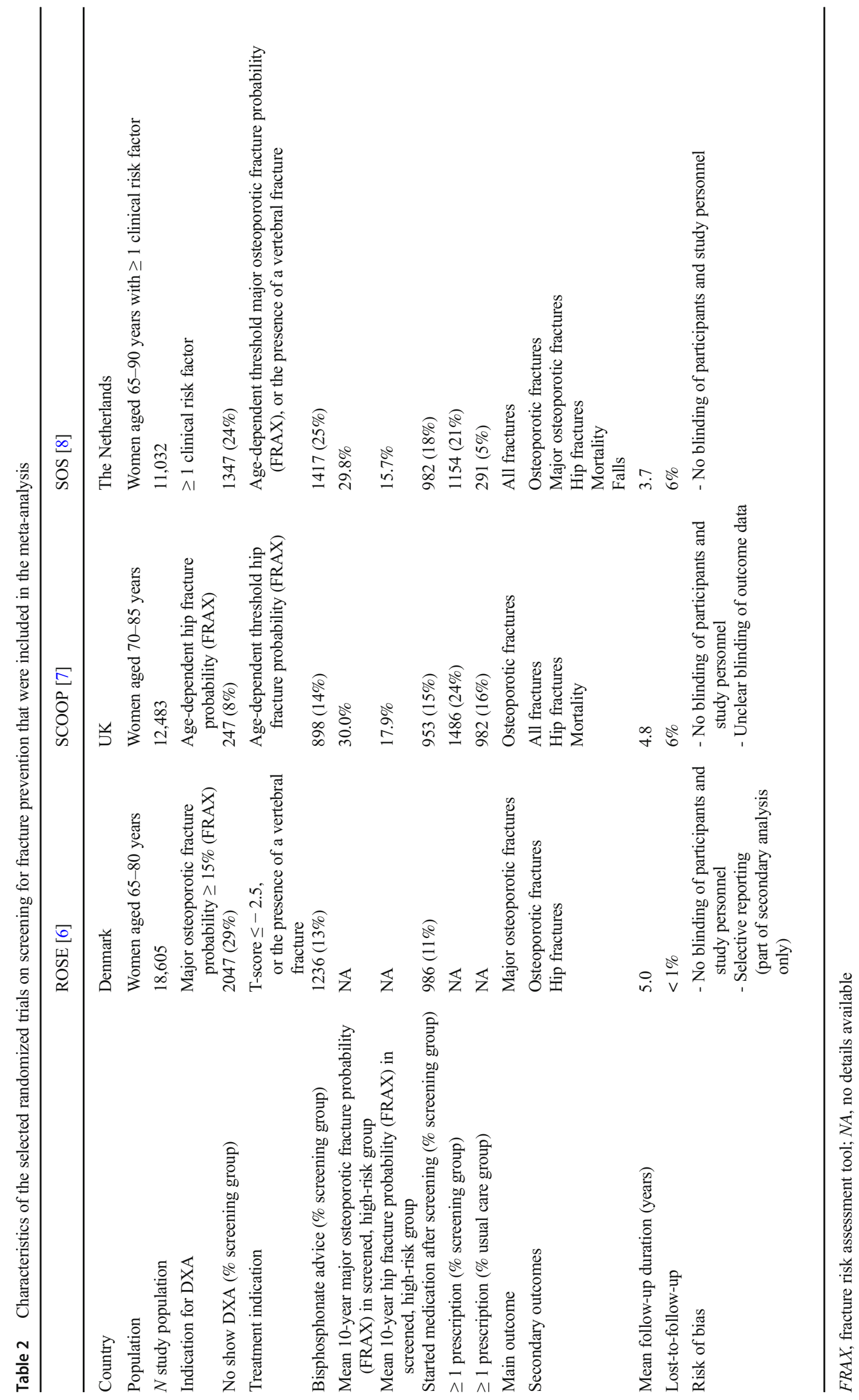


Characteristics of the studies are presented in Table 2. All studies were performed among older women and used a combination of clinical risk factors and bone densitometry to screen participants for a high fracture risk. In the ROSE study, the SCOOP study, and the SOS, respectively, $11 \%, 15 \%$, and $18 \%$ of the participants in the intervention group started medication after screening. The risk of bias assessment showed that all three studies were not able to blind the participants and study personnel, and unclear blinding of outcome data by the SCOOP study [12].

In the ROSE study, the intention to treat population included all women who were mailed a questionnaire. We used the data from the first per protocol analysis in the meta-analysis, thereby including all participants who returned the questionnaire with adequate information to calculate a baseline fracture probability. These data were most comparable with the intention-to-treat data from the SOS and the SCOOP study. All three studies used the fracture risk assessment tool (FRAX) in combination with DXA as screening strategy. Between-study heterogeneity was low $\left(I^{2}<0.5\right.$ for all outcomes).

Figure 2 shows forest plots for the different outcomes. The pooled HR for all fractures was $0.95(95 \% \mathrm{CI}=0.89-1.02)$. Screening led to a significantly lower risk of osteoporotic fractures ( $\mathrm{HR}=0.95,95 \% \mathrm{CI}=0.89-1.00)$, major osteoporotic fractures $(\mathrm{HR}=0.91,95 \% \mathrm{CI}=0.84-0.98)$, and hip fractures $(\mathrm{HR}=0.80,95 \% \mathrm{CI}=0.71-0.91)$. The number needed to screen, starting with the completion of a complete questionnaire, was 247 for an osteoporotic fracture and 272 for a hip fracture. A bone densitometry was performed in $46 \%$ of these women, corresponding to 113 bone densitometry examinations to prevent one osteoporotic fracture, and 124 to prevent one hip fracture. The number needed to treat based on the women in the group with an increased fracture risk who started anti-osteoporosis medication was 25 for an osteoporotic fracture and 28 for a hip fracture.

The pooled HR for the secondary outcome all-cause mortality was 1.04 (95\% CI $=0.95-1.14)$. Post-hoc sensitivity analysis on hip fractures that excluded the findings from the SCOOP study showed a HR or $0.85(95 \% \mathrm{CI}=$ $0.73-1.00)$.

\section{Discussion}

This systematic review and meta-analysis with data from over 42,000 participants showed that screening for high fracture risk and subsequent treatment in older women reduced all osteoporotic fractures, and hip fractures in particular. The observed relative risk reductions gradually increased according to the fracture type (5\% for osteoporotic fractures, $9 \%$ for major osteoporotic fractures, and $20 \%$ for hip fractures), with a larger reduction for fractures more related to osteoporosis and therefore more responsive to treatment. No associations were observed for the secondary outcome all-cause mortality. Taking into account that only $11-18 \%$ of the participants in the intervention groups were advised to use anti-osteoporosis medication; the observed relative fracture risk reductions are substantial. The low number needed to screen and number needed to treat emphasize the clinical relevance of screening as intervention to reduce hip fractures.

The observed number needed to treat is comparable with that reported in drug trials of anti-osteoporosis medication; 20-200 patients would need to be treated with alendronate for 5 years to prevent one fracture, depending on the a priori fracture risk and type of fracture [4]. Compared with other population screening programs, the number needed to screen is acceptable if one takes into account that the mortality after a hip fracture is substantial [20]. For instance, by breast cancer screening, 12.5 deaths are reduced by screening, 10,000 women aged 50 to 69 years for 10 years (number needed to screen $=800$ ) [21]. In addition, for colorectal screening, the numbers needed to screen were 377 (95\% CI, 249-887) and 864 (95\% CI, 672-1266) for guaiac fecal occult blood testing and flexible sigmoidoscopy screening, respectively [22].

The three included studies all had a pragmatic design. All three used a preselection of participants before bone densitometry, based on a FRAX calculation or the presence of clinical risk factors. An important difference in selection for treatment was the use of vertebral fracture assessment in ROSE and SOS that was absent in SCOOP. Our meta-analysis was limited to the studies that used at least bone densitometry as screening instrument. The effects of other screening approaches were not considered. One screening trial that was excluded in this meta-analysis because of the absence of bone densitometry used only thoracolumbar radiographs. In that study, the odds ratio for new fractures was $0.60(95 \% \mathrm{CI}=0.35-1.03)$ after a short follow-up of 12 months. Although this study did not reach statistical significance, it also supports the value of vertebral fracture assessment in addition to bone densitometry [17].

While the strategies and the overall results were consistent between the included studies, which is also reflected in low between-study heterogeneity, a distinct significant relative risk reduction of hip fractures of $28 \%$ has been observed in the SCOOP study [12]. Although the SCOOP study used the FRAX probability for hip fractures rather than the FRAX probabilities for major osteoporotic fractures, the questions was raised if this reduction of hip fractures could be explained by the intervention with medication alone [23]. It is furthermore unlikely that the strong effect on hip fractures was not followed by a reduction in osteoporotic fractures in the SCOOP study. We, therefore, performed a sensitivity analysis on hip fractures without the findings from the SCOOP study. The hip fracture reduction was still $15 \%$ and remained statistically significant. 
Fig 2 Forest plots of screening for fracture prevention versus usual primary care, regarding fracture type (a-d) and all-cause mortality (e). Note: From the ROSE study, the data from the first per protocol analysis were used, thereby including all participants with baseline FRAX data. These data were most comparable to the data from the SCOOP study and the SOS, with data from all participants with respective baseline questionnaire data or $\geq 1$ clinical risk factors for fractures a All fractures

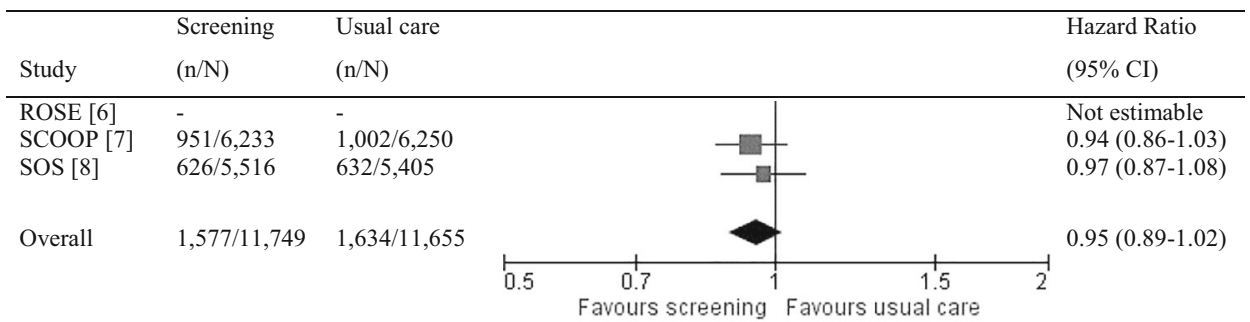

b Osteoporotic fractures

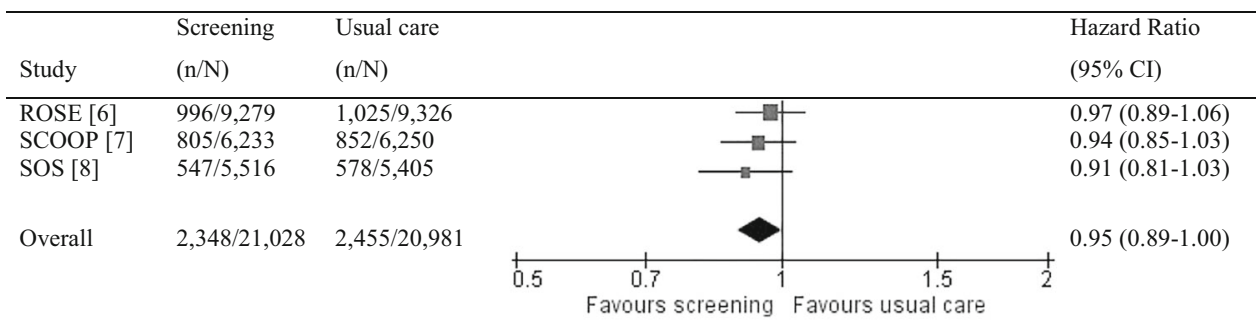

C Major osteoporotic fractures

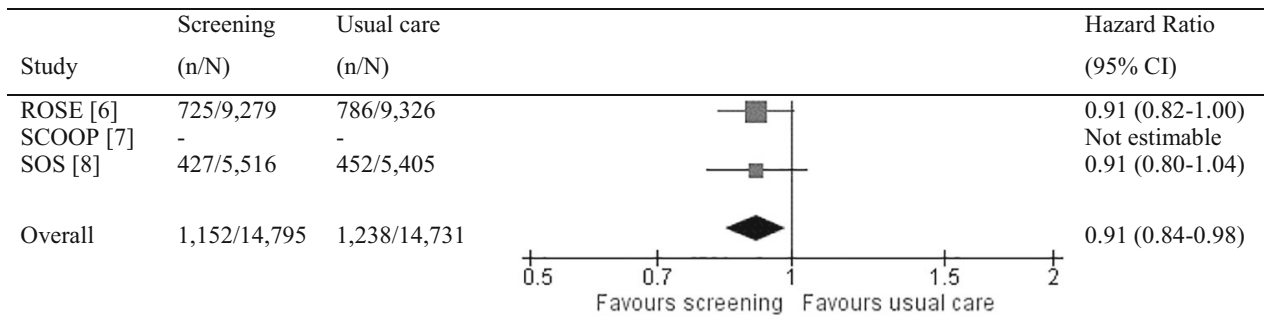

d Hip fractures

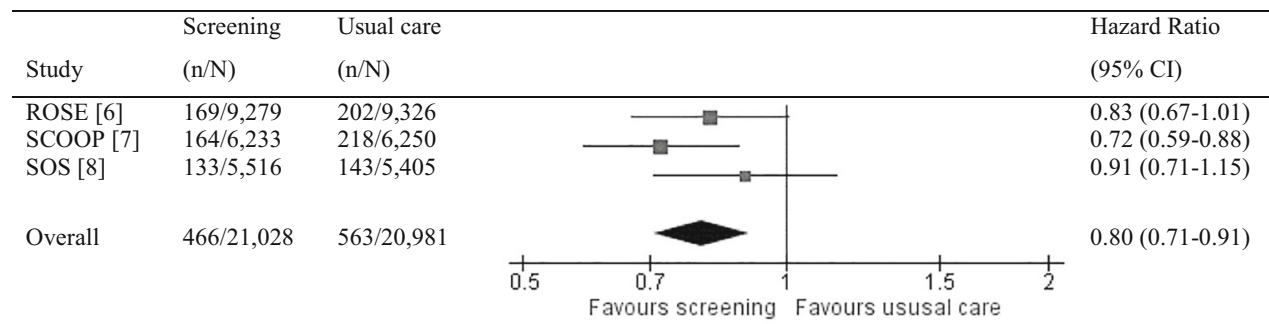

e All-cause mortality

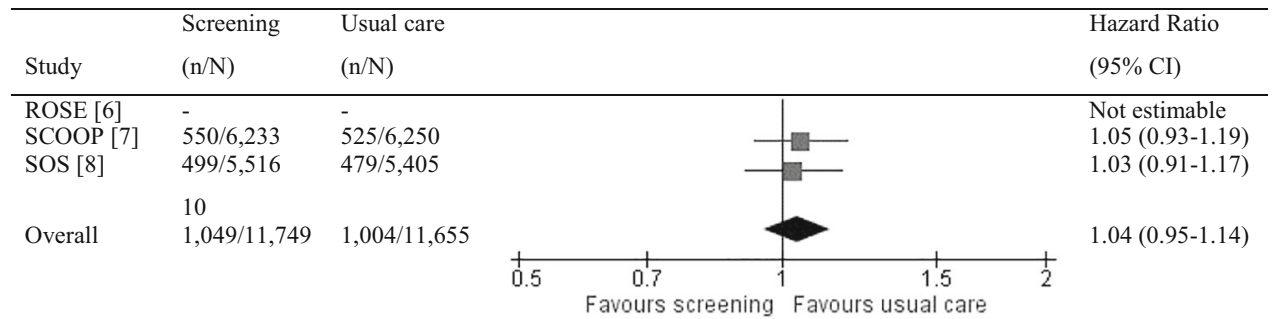

The participation rates to the screening programs in the three studies ranged from 34 to $61 \%$ and an additional 8 to $25 \%$ of the participants dropped out before examination. In the ROSE study and the SOS, there was a relatively high dropout rate before examination in the screening groups, whereas in the SCOOP study, the participation rate was lower. Moreover, during follow-up, the difference in received treatment between the screening groups and the usual care groups was reduced by non-adherence and an increase of usual care prescriptions in the control group due to the non-blinded 
design. This treatment contrast declined to $3.5-8 \%$ after 3.7 to 5 years. The optimal follow-up duration is unclear, but the chosen periods seem to be a reasonable choice between the time to the effect of medication and the decline of the treatment contrast.

Some limitations of this study should be mentioned. Only three studies were selected for inclusion after systematic review. Although the total number of participants was as much as 42,000 , not all outcomes were reported by all the three studies resulting in lower numbers depending on the outcome. The included studies were of high quality, but complete blinding was not feasible due to the nature of the intervention. From ROSE, the results from the participants with baseline FRAX data were pooled to reach a population that was comparable to the intention to treat populations of SCOOP and SOS. Starting from there, all participants were followed, irrespective of drop-out status, treatment prescription, or medication adherence. This approach is the best way to prevent a false-positive conclusion about the effect of the intervention due to the selection bias and provides real-life estimates. The effect of screening and treatment in the participants who have actually undergone the intervention is likely to be underestimated.

Recently, the need for BMD and fracture risk assessment before the initiation of anti-osteoporosis medication has been questioned. A recent randomized controlled trial that examined the effect of 5-mg zoledronate infusions at 18 months interval in women with osteopenia observed that fracture rates were lower in the intervention than the placebo group, and that, this effect was consistent across a wide range of baseline characteristics [24, 25]. While interpreting these important findings and its implications for osteoporosis management strategies, the cost-effectiveness should be considered as well. In contrast to population screening for which the current metaanalysis showed that hip fractures can be prevented, no effect on hip fractures was observed by applying zoledronate infusions to osteopenic women, mainly because the occurrence of hip fractures was minimal in this group [25]. It is unlikely that a fracture reducing intervention is cost-effective if it does not lead to a reduction of hip fractures. Therefore, the costeffectiveness of different strategies and thresholds for inclusion needs further investigation.

The current findings indicate that screening in primary care is a serious option to reduce fractures and should be considered by policymakers and guideline committees. Subgroup analysis and comparison of the different strategies could provide valuable information about the most effective and costeffective strategy for the implementation of population screening. To make screening programs even more effective, we think that further research should focus on ways to increase participation to the program and adherence to medication.

In conclusion, this is the first fracture risk screening study that showed a reduction of osteoporotic fractures and hip fractures with a high level of evidence. Screening for high fracture risk in primary care should be considered as evidence-based, way to reduce osteoporotic fractures.

Funding information The authors declare no support from any organization for the submitted work (TM, KS, HvdH, CN, PE); financial relationships with Stichting Achmea Gezondheidszorg, Achmea, VGZ Zorgverzekeraar, and Stichting Artsen Laboratorium en Trombosedienst during the conduct of the SALT Osteoporosis Study (TM, KS, CN, PE); no other relationships or activities that could appear to have influenced the submitted work (TM, KS, HvdH, CN, PE).

\section{Compliance with ethical standards}

Conflicts of interest None

Ethical approval and informed consent The manuscript does not contain patient data.

Open Access This article is distributed under the terms of the Creative Commons Attribution-NonCommercial 4.0 International License (http:// creativecommons.org/licenses/by-nc/4.0/), which permits any noncommercial use, distribution, and reproduction in any medium, provided you give appropriate credit to the original author(s) and the source, provide a link to the Creative Commons license, and indicate if changes were made.

\section{References}

1. Dunnewind T, Dvortsin EP, Smeets HM, Konijn RM, Bos JHJ, de Boer PT, van den Bergh JP, Postma MJ (2017) Economic consequences and potentially preventable costs related to osteoporosis in the Netherlands. Value Health 20:762-768

2. Hernlund E, Svedbom A, Ivergard M, Compston J, Cooper C, Stenmark J, McCloskey EV, Jonsson B, Kanis JA (2013) Osteoporosis in the European Union: medical management, epidemiology and economic burden. A report prepared in collaboration with the International Osteoporosis Foundation (IOF) and the European Federation of Pharmaceutical Industry Associations (EFPIA). Arch Osteoporos 8:136

3. Wells G, Cranney A, Peterson J, Boucher M, Shea B, Robinson V, Coyle D, Tugwell P (2008) Risedronate for the primary and secondary prevention of osteoporotic fractures in postmenopausal women. Cochrane Database Syst Rev CD004523

4. Wells GA, Cranney A, Peterson J, Boucher M, Shea B, Robinson V, Coyle D, Tugwell P (2008) Alendronate for the primary and secondary prevention of osteoporotic fractures in postmenopausal women. Cochrane Database Syst Rev CD001155

5. Kanis JA, Cooper C, Rizzoli R, Reginster JY (2019) European guidance for the diagnosis and management of osteoporosis in postmenopausal women. Osteoporos Int 30:3-44

6. Papaioannou A, Morin S, Cheung AM et al (2010) 2010 clinical practice guidelines for the diagnosis and management of osteoporosis in Canada: summary. Can Med Assoc J 182:1864-1873

7. Curry SJ, Krist AH, Owens DK et al (2018) Screening for osteoporosis to prevent fractures: us preventive services task force recommendation statement. Jama 319:2521-2531

8. Elders PJM, Dinant GJ, van Geel T, Maartens LWF, Merlijn T, Geijer RMM, Geraets JJXR (2012) NHG-Standaard Fractuurpreventie (tweede herziening). Huisarts Wet 55:452-458 
9. Peto L, Allaby M (2013) Screening for osteoporosis in postmenopausal women: a report for the UK National Screening Committee. Solution for public health, Oxford

10. Merlijn T, Swart KMA, Van Schoor NM, et al. (2019) The effect of a screening and treatment program for the prevention of fractures in older women: a randomized pragmatic trial. J Bone Min Res Accepted

11. Rubin KH, Rothmann MJ, Holmberg Tet al (2018) Effectiveness of a two-step population-based osteoporosis screening program using FRAX: the randomized Risk-stratified Osteoporosis Strategy Evaluation (ROSE) study. Osteoporos Int 29:567-578

12. Shepstone L, Lenaghan E, Cooper C et al (2018) Screening in the community to reduce fractures in older women (SCOOP): a randomised controlled trial. Lancet 391:741-747

13. Higgins J, Green S (2015) Cochrane handbook for systematic reviews of interventions. The Cochrane Collaboration

14. Buist DS, LaCroix AZ, Manfredonia D, Abbott T (2002) Identifying postmenopausal women at high risk of fracture in populations: a comparison of three strategies. J Am Geriatr Soc 50: 1031-1038

15. Lacroix AZ, Buist DS, Brenneman SK, Abbott TA 3rd (2005) Evaluation of three population-based strategies for fracture prevention: results of the osteoporosis population-based risk assessment (OPRA) trial. Med Care 43:293-302

16. Barr RJ, Stewart A, Torgerson DJ, Seymour DG, Reid DM (2005) Screening elderly women for risk of future fractures-participation rates and impact on incidence of falls and fractures. Calcif Tissue Int $76: 243-248$

17. Clark EM, Gould V, Morrison L, Ades AE, Dieppe P, Tobias JH (2012) Randomized controlled trial of a primary care-based screening program to identify older women with prevalent osteoporotic vertebral fractures: Cohort for Skeletal Health in Bristol and Avon (COSHIBA). J Bone Miner Res 27:664-671

18. Barr RJ, Stewart A, Torgerson DJ, Reid DM (2010) Population screening for osteoporosis risk: a randomised control trial of medication use and fracture risk. Osteoporos Int 21:561-568
19. Schneider DL, Worley K, Beard MK, Iannini M, Ko M, McCallum J, Pulicharam R, Steinbuch M (2010) The primary care osteoporosis risk of fracture screening (POROS) study: design and baseline characteristics. Contemp Clin Trials 31:336-344

20. Katsoulis M, Benetou V, Karapetyan T, Feskanich D, Grodstein F, Pettersson-Kymmer U, Eriksson S, Wilsgaard T, Jørgensen L, Ahmed LA, Schöttker B, Brenner H, Bellavia A, Wolk A, Kubinova R, Stegeman B, Bobak M, Boffetta P, Trichopoulou A (2017) Excess mortality after hip fracture in elderly persons from Europe and the USA: the CHANCES project. J Intern Med 281: 300-310

21. Nelson HD, Fu R, Cantor A, Pappas M, Daeges M, Humphrey L (2016) Effectiveness of breast cancer screening: systematic review and meta-analysis to update the 2009 US PREVENTIVE SERVICES TASK FORCE RECOMMENDATION. Ann Intern Med 164:244-255

22. Fitzpatrick-Lewis D, Ali MU, Warren R, Kenny M, Sherifali D, Raina P (2016) Screening for Colorectal Cancer: A Systematic Review and Meta-Analysis. Clin Colorectal Cancer 15:298-313

23. Swart KMA, Merlijn T, Netelenbos JC, Elders PJM (2018) A closer look at SCOOP: screening for fracture prevention. Lancet 392:552

24. Reid IR, Horne AM, Mihov B, Stewart A, Garratt E, Wiessing KR, Bolland MJ, Bastin S, Gamble GD (2019) Anti-fracture efficacy of zoledronate in subgroups of osteopenic postmenopausal women: secondary analysis of a randomized controlled trial. J Intern Med

25. Reid IR, Horne AM, Mihov B, Stewart A, Garratt E, Wong S, Wiessing KR, Bolland MJ, Bastin S, Gamble GD (2018) Fracture prevention with zoledronate in older women with osteopenia. $\mathrm{N}$ Engl J Med 379:2407-2416

Publisher's note Springer Nature remains neutral with regard to jurisdictional claims in published maps and institutional affiliations. 